\title{
DOENCAS DE RUMINANTES DOMÉSTICOS DIAGNOSTICADAS NO LABORATÓRIO DE PATOLOGIA VETERINÁRIA DA UNIVERSIDADE FEDERAL DO PARANÁ: 1075 CASOS
}

(Desease of domestic ruminants diagnosed in Veterinary Pathology Laboratory of the Federal University of Paraná: 1075 cases)

Lew Kan Sprenger, Larissa Wünsche Risolia, Lorran Baeumle Gabardo, Marcelo Beltrão Molento, Antônio Waldir Cunha da Silva, Renato Silva de Sousa ${ }^{1}$

${ }^{1}$ Correspondência: renatosousa@ufpr.br

RESUMO: O objetivo do estudo foi determinar a prevalência das doenças encontradas em bovinos, caprinos e ovinos examinados pelo Laboratório de Patologia Veterinária da Universidade Federal do Paraná (LABDP-UFPR), Curitiba. Foram pesquisados os protocolos de necropsia dos animais, no período de fevereiro de 1967 a dezembro de 2012. Foram examinados 1075 cadáveres de ruminantes domésticos no LABPV-UFPR, sendo que 59,9\% (644/1075) eram bovinos, 23,6\% (254/1075) de ovinos e $16,5 \%(177 / 1075)$ de caprinos. A tuberculose foi a principal causa de morte em bovinos 8,23\% (53/644), seguido de raiva $7,30 \%(47 / 644)$, tristeza parasitária bovina $6,68 \%(43 / 644)$ e intoxicação por Pteridium aquilinum 6,06\% (39/644). A hemoncose foi a principal causa de morte em ovinos 20,87\% (53/254), seguido de enterotoxemia 11,02\% (28/254), intoxicação por Ipomoea asarifolia 7,48\% (19/254) e tétano 7,48\% (19/254). A hemoncose foi o maior responsável pela mortalidade de caprinos $17,51 \%(31 / 177)$, seguido de artrite- encefalite caprina (CAE) 7,91\% (14/177), tétano 6,21\% (11/177) e fasciolose $4,52 \%$ (8/177). Os dados presentes neste trabalho permitem que veterinários tenham acesso as principais enfermidades que acometem os ruminantes domésticos na Região Sudeste do Paraná.

Palavras-chave: Bovinos; caprinos; histopatologia; ovinos

ABSTRACT: The aim of the study was to determinated the prevalence of deseases in cattle, goat and sheeps examined by Veterinary Pathology Laboratory, Federal University of Paraná (LABDP-UFPR), Curitiba. Necropsy protocols of the animals were surveyed, from February 1967 to December 2012. Were examined in LABPVUFPR 1075 cases. Of the total, 59,9\% (644/1075) were cattle, 23,6\% (254/1075) sheep and $16,5 \%(177 / 1075)$ of goats. Tuberculosis was the most prevalent cause of death in cattle $8.23 \%$ (53/644), followed by anger $7.30 \%(47 / 644), 6.68 \%$ bovine tick fever (43/644) and Pteridium aquilinum poisoning 6.06\% (39/644). The hemoncose was the leading in sheep $20.87 \%$ (53/254), followed by Clostridium perfringens enterotoxemia $11.02 \%$ (28/254), Ipomoea asarifolia poisoning $7.48 \%$ (19/254), and tetanus $748 \%(19 / 254)$. The hemoncose was largely responsible for the mortality of goats $17.51 \%(31 / 177)$, followed by caprine arthrite encephalitis virus $7.91 \%(14 / 177)$, tetanus $6.21 \%(11 / 177)$ and fascioliasis $4.52 \%(8 / 177)$. Data presented in this work, provides scientific information to veterinarians have access to the main diseases that affect domestic ruminants in Southeastern Paraná.

Key Words: Cattle; goat; histopathology; sheep 


\section{INTRODUÇÃO}

Atualmente o Brasil é um dos maiores produtores e exportadores de carne, de diversos animais, no mundo (Pontes e Lima; 2013). Entre as regiões brasileiras, a Sul é a grande produtora desses produtos e derivados (Dos Anjos et al. 2010). O país tem um dos maiores rebanhos comerciais de bovinos do planeta, tendo a bovinocultura como carro-chefe da pecuária nacional (Almeida et al. 2012). Todavia, existem outras produções animais que também trazem grandes divisas a balança comercial. O rebanho caprino brasileiro é estimado em 9,5 milhões, representando assim uma outra importante atividade pecuária no país (Costa et al. 2011). Por fim, o mercado de ovinos está em franco crescimento, movimentando milhões de reais no mercado pecuário nacional, motivado, entre outros fatores, pelo aumento considerável do consumo da carne, ano após ano (Taparo et al. 2012).

Contudo, muitas perdas ocorrem causadas por enfermidades, acarretando em perdas econômicas que muitas vezes podem inviabilizar a criação. Logo, o conhecimento das características epidemiológicas dessas doenças, em determinada localidade, é uma ferramenta útil para maximizar o lucro. Mesmo sendo difícil a realização da necropsia para determinar a causa da morte dos animais, esta é uma ferramenta diagnóstica muito importante para os profissionais (McGavin e Zachary, 2009). Muitas doenças são facilmente confundíveis, se for realizado apenas o diagnóstico clínico e diversas outras podem não ser descobertas, se o diagnóstico laboratorial não for conclusivo (Santos e Alessi, 2010).

Sendo assim, o estudo retrospectivo baseado na coleta de dados armazenados por instituições de pesquisa é de suma importância na epidemiologia e patologia veterinária
(Lucena et al. 2010). Com essas informações os médicos veterinários podem aumentar a sua precisão diagnóstica, conhecer a prevalência das enfermidades da região, definir estratégias de controle das enfermidades baseando-se no histórico sanitário (Fighera, 2008). Porém poucos são os estudos retrospectivos encontrados na literatura (Fighera et al. 2008b; Sprenger et al. 2014).

O objetivo do presente estudo foi determinar a frequência e características epidemiológicas das doenças dos ruminantes domésticos examinados no Laboratório de Patologia Veterinária da Universidade Federal do Paraná (LABDP-UFPR).

\section{MATERIAL E MÉTODOS}

Para a produção do banco de dados utilizados no presente trabalho, foram analisados os protocolos referentes as necropsias de bovinos, caprinos e ovinos, realizados pelo LABPV-UFPR, entre fevereiro de 1967 a dezembro de 2012. Foram coletadas as informações quanto à espécie, ao sexo, à idade e à causa de óbito. Coletaramse todas as informações sobre as doenças e causas iniciais do processo que conduziu a morte dos indivíduos. Embora muitos destes tivessem mais do que um processo patológico, um único diagnóstico definitivo foi estabelecido para cada caso, sendo semelhante ao que foi realizado em outros estudos (Moore et al. 2001; Bonnett et al., 2005; Fighera et al., 2008). Quando não continham informações suficientes para se chegar a um diagnóstico conclusivo, os casos foram denominados inconclusivos. Quanto ao sexo, os animais foram divididos apenas em machos e fêmeas, independentemente de serem castrados. Já para a qualificação a respeito da idade, utilizouse os critérios descritos a seguir. 
Bovinos: menores de 18 meses; $18-60$ meses; 60 meses ou mais. Caprinos: menores que 12 meses; 12-42 meses; maiores que 42 meses. Ovinos: menores que 12 meses; $12-42$ meses; maiores que 42 meses.

As doenças dos animais foram divididas por grupos, segundo critério adaptado de Lucena et al.(2010) em: distúrbios causados por agentes físicos, distúrbios do desenvolvimento, distúrbios iatrogênicos, distúrbios imunogênicos, doenças degenerativas, doenças infecciosas, doenças parasitárias, doenças metabólicas e nutricionais, intoxicações e toxiinfecções, neoplasmas, outros distúrbios e causa desconhecida.

Por fim, foi calculada a prevalência das enfermidades utilizando o programa Epi Info® versão 3.5.1.

\section{RESULTADOS E DISCUSSÃO}

Durante o período de fevereiro de 1967 a dezembro de 2012, foram examinados 1075 cadáveres de ruminantes domésticos no LABPVUFPR, sendo que $59,9 \%$ (644/1075) eram bovinos, $23,6 \%(254 / 1075)$ de ovinos e 16,5\% (177/1075) de caprinos. A proporção de bovinos examinados foi estatisticamente maior que as demais espécies $(p<0,05)$. Em bovinos, 87,0\% (560/644) dos animais acometidos eram fêmeas, $12,7 \%$ (82/644) machos e 0,3\% (2/644) não informado. Esta maior proporção de fêmeas, deve-se ao alto número de animais da raça holandesa examinados. Em ovinos, 63,8\% (162/254) dos animais acometidos eram fêmeas, 34,6\% (88/254) machos e 1,6\% (4/254) não informado. Em caprinos, $65,5 \%$ (116/172) dos animais acometidos eram fêmeas e $34,5 \%$ (61/172) machos. Para as duas últimas espécies mencionadas, 0 alto número de fêmeas é justificado pelas características de criação, onde os machos são abatidos mais cedo e as propriedades possuem um número de matrizes superior ao de reprodutores.

Em bovinos, os grupos de enfermidades mais diagnosticadas foram, doenças infecciosas $24,84 \%$ (160/644), seguidas de doenças parasitárias $\quad 24,07 \% \quad(155 / 644)$, intoxicações $11,49 \% \quad(74 / 644) \quad$ e neoplasmas 9,94\% (64/644) (Quadro 1). As duas primeiras categorias em conjunto possuem alta porcentagem nas causas de mortes, 48,9\% (315/644), principalmente devido as falhas de manejo sanitário existentes na bovinocultura paranaense (Dias et al. 2008). Sendo este um problema nacional (Almeida e Michels, 2012). Em países onde os programas de sanidade animal estão melhor implementados, estes motivos de mortalidade são os primeiros igualmente, contudo possuem uma menor importância (Dutra e Pereira, 2012). Os casos de intoxicações devem-se, principalmente, a falta de cuidado dos produtores em não retirar da pastagem plantas tóxicas como Pteridium aquilinum e também em aplicar incorretamente medicamentos antiparasitários, como organofosforados. A alta prevalência de neoplasias ocorreu devido à alta faixa etária dos animais envolvidas no estudo, 51,4 meses. A idade crítica para ocorrência de neoplasias nesta espécie animal é a partir de 40 meses.

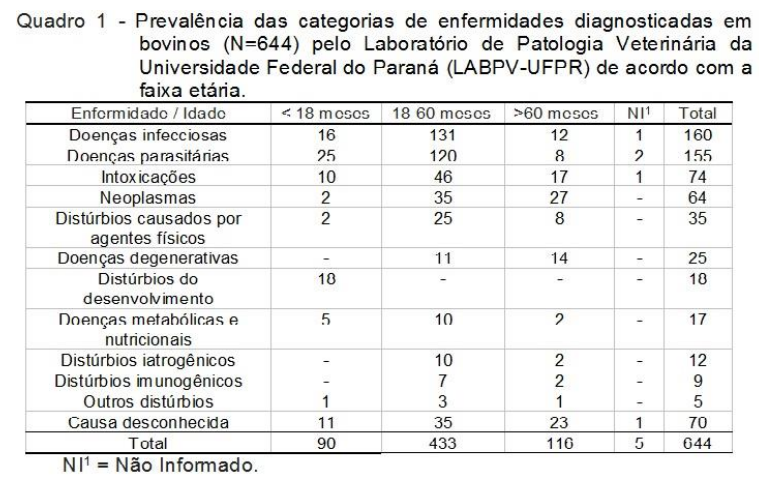

A tuberculose foi a principal causa de morte em bovinos $8,23 \%$ 
(53/644), seguido de raiva $7,30 \%$ $(47 / 644)$, tristeza parasitária bovina $6,68 \%$ (43/644) e intoxicação por Pteridium aquilinum 6,06\% (39/644) (Quadro 2). Esse resultado foi semelhante ao trabalho realizado por Lucena et al., 2010, em um estudo realizado em Santa Maria, Rio Grande do Sul. Mesmo a tuberculose sendo a principal enfermidade diagnosticada no período analisado, esta não foi a causa direta de mortalidade dos animais. A doença está difundida por grande extensão do território nacional, tendo grande importância em saúde pública, tendo em vista que é uma das principais zoonoses bacterianas existentes (Pacheco et al., 2009). No Brasil, há cerca de 1,4 milhões de óbitos de humanos por tuberculose (WHO, 2012). A raiva bovina é uma doença infecciosa fatal, causada por vírus do gênero Lyssavirus, que gera grandes problemas econômicos a pecuária nacional. Apesar de existirem levantamentos anuais apontando o número de casos, os dados são subestimados devido à falta de notificação pelos produtores (BRASIL, 2010). Apesar de serem poucos os relatos de transmissão por bovinos, destaca-se a importância dos casos devido a indicação de fontes transmissoras da enfermidade (Lima \& Gagliani, 2014). O complexo Tristeza Parasitária Bovina, é um dos principais causadores de prejuízos na bovinocultura nacional, sendo endêmica em muitas regiões do país e transmitida apenas pelo carrapato Rhipicephalus (Boophilus) microplus (Gonçalves et al. 2011). Em pesquisa realizada em Pelotas, Rio Grande do Sul, foi observada prevalência de $7,83 \%$ (374/4775) (Tortelli et al., 2005). Analisando amostras de animais provenientes da Região Sudoeste do Rio Grande do Sul, observou-se infecção em 4,7\% (231/4884) (Almeida et al. 2006). Este valores são semelhantes ao encontrado no presente estudo, 6,68\% (43/644). A ingestão de Pteridium aquilinum, conhecida popularmente como samambaia, em

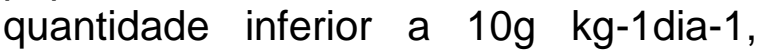
durante um ou mais anos pode causar hematúria enzoótica bovina (Tokarnia et al., 2000). Esta é a principal planta tóxica encontrada na Região Sul, devido principalmente ao solo ácido do local (Pessoa et al. 2013). A ingestão de plantas tóxicas é uma das principais causas de óbito na referida localidade, como demonstrado em outros estudos (Riet-Correa e Medeiros, 2001; Lucena et al., 2010).

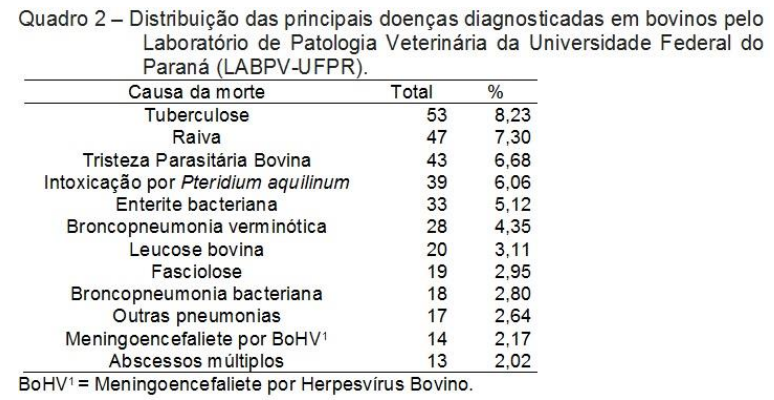

Em ovinos, os grupos de enfermidades mais prevalentes foram, doenças parasitárias 28,35\% (72/254), seguidas de doenças infecciosas $23,23 \%$ (59/254), intoxicações 8,66\% (22/254) e doenças metabólicas e nutricionais 3,94\% (10/254) (Quadro 3). Estes resultados diferem dos dados encontrados em estudo realizado por Rissi et al., 2010, onde as intoxicações e toxi-infecções obtiverem maior relevância na mortalidade dos animais, devido principalmente aos casos de ingestão de altas doses de Senecio sp. e cobre. As doenças infecciosas e parasitárias, em conjunto, foram a principal causa de mortalidade dos animais $51,6 \%$ (131/254). O impacto causado por estas enfermidades não reduzirá enquanto não forem planejadas, implementadas e fiscalizadas medidas adequadas de manejo sanitário nas propriedades pecuárias (Silva et al., 2013). Estudos apontam que a realização de medidas 
básicas de prevenção a doenças infectocontagiosas, podem diminuir a sua incidência consideravelmente (Thrusfield, 1995). Os casos de intoxicações ocorreram principalmente pela ingestão de Ipomoea asarifolia 86,4\% (19/22). Estima-se que anualmente morrem de intoxicação por plantas de 399.800 a 445.309 ovinos (Pessoa et al. 2013). As doenças metabólicas e nutricionais decorreram da falta de alimento em períodos críticos de seca e suas consequências indiretas. Isso ocorre porque a ingestão de matéria seca pode se tornar limitada em épocas na qual a forrageira disponível apresenta baixa qualidade nutricional ou disponibilidade. Associando este quadro, há a menor capacidade física do trato gastrointestinal de animais jovens, fazendo com que estes não ingiram a quantidade de nutrientes necessários diariamente, tornando-os mais suscetíveis (Cannas et al., 2004).

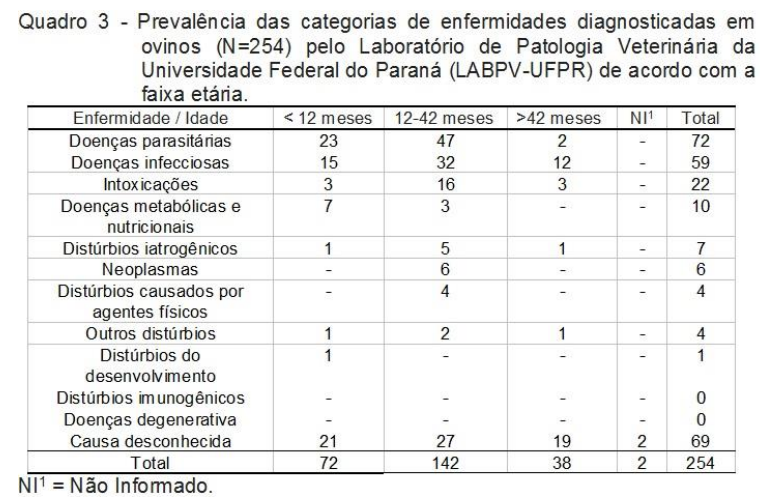

A hemoncose foi a principal causa de morte em ovinos 20,87\% (53/254), seguido de enterotoxemia $11,02 \%$ (28/254), intoxicação por Ipomoea asarifolia 7,48\% (19/254) e tétano 7,48\% (19/254) (Quadro 4). Esse resultado foi semelhante ao trabalho realizado por Lucena et al. 2010, em um estudo realizado em Santa Maria. O Haemonchus contortus é o principal parasita de pequenos ruminantes, sendo também o principal responsável por mortalidade, em muitas regiões do mundo (Fortes et al., 2013), incluindo a
Região Sul brasileira (Sprenger et al. 2013). A enterotoxemia, em ovinos, geralmente é hiperaguda e não há sinais clínicos. Na necropsia, se observa hiperemia do intestino delgado, rins amolecidos, hemorragias nas serosas e líquidos em cavidades (Layana et al., 2006). Animais jovens com bons níveis nutricionais, como o que ocorreu neste estudo, onde $53,6 \%(15 / 28)$ dos casos ocorreram em animais com menos de 12 meses de idade, normalmente são acometidos pela forma hiperaguda da doença. A Ipomoea asarifolia é uma planta mais comum no Nordeste do país, contudo existem casos de intoxicação nos estados do Sul e também no Uruguai (Riet-Correa e Medeiros, 2001). Apesar de ser uma planta pouco palatável, em condições de pouca disponibilidade de alimentos, os animais tendem a ingerir, devido ao aumento gradual da fome (Riet-Correa et al. 2011). Os casos de tétano geralmente ocorrem após castração, caudectomia, descorna, ou parto (Guedes et al., 2007). No presente estudo, 78,94\% (15/19) dos casos ocorreram após o parto.

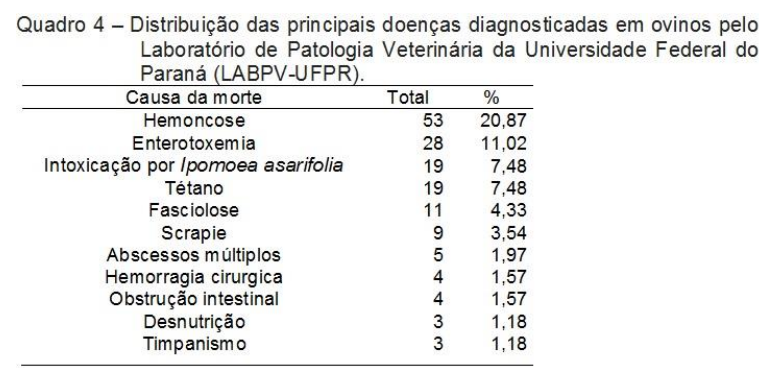

Em caprinos, os grupos de enfermidades mais prevalentes foram, doenças parasitárias 24,29\% (43/177), seguidas de doenças infecciosas $18,64 \%$ (33/177), doenças metabólicas e nutricionais $9,03 \% \quad(16 / 177)$ e intoxicações 9,03\% (16/177) (Quadro 5). Novamente, as doenças infecciosas e parasitárias são a maior causa de mortalidade dos animais $42,9 \%$ (76/177). Este grupo de moléstias são as que causam maior prejuízo na 
caprinocultura mundial, devido a facilidade de disseminação, apoiada pela falta de práticas de manejo sanitário implantadas (Thrusfield, 1985; Ezebuiro et al., 2008). No presente trabalho, doenças metabólicas e nutricionais foram 0 terceiro maior motivo de mortalidade dos caprinos $9 \%$ $(16 / 177)$. Este resultado também foi semelhante ao observado por Rosa et al. 2013, que analisou um banco com dados de necropsia composto por 114 animais. As intoxicações decorreram de diversos motivos, como intoxicação por cobre 12,5\% (2/16), intoxicação por Brachiaria brizantia 12,5\% (2/16), intoxicação por Senecio sp. 12,5\% $(2 / 16)$, intoxicação por uréia $12,5 \%$ $(2 / 16)$, entre outras causas. No Brasil, estima-se que a mortalidade por plantas tóxicas, varia entre $6,4 \%$ a $7,69 \%$ do rebanho caprino (Assis et al., 2010).

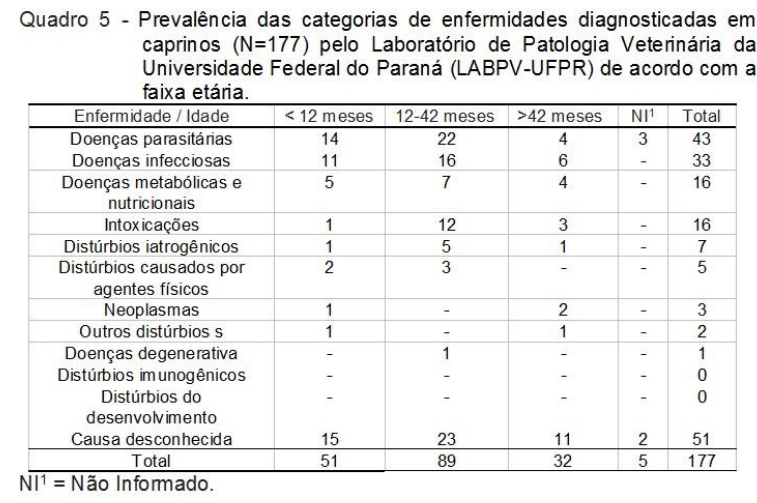

A hemoncose foi o maior responsável pela mortalidade de caprinos $17,51 \%$ (31/177), seguido de artrite- encefalite caprina (CAE) 7,91\% (14/177), tétano $6,21 \%$ (11/177) e fasciolose 4,52\% (8/177) (Quadro 6). Este resultado difere do encontrado por Rosa et al. 2013, que observaram que as principais causas de mortalidade foram haemoncose, listeriose, eimeriose e intoxicação por closantel. $\mathrm{O} H$. contortus, assim como para ovinos, é o principal endoparasita responsável por mortalidade em caprinos (Molento et al. 2013). Dos animais com CAE, 14,29\% (2/14) morreram de leucoencefalomielite e eram animais menores de 12 meses e $85,71 \%$ (12/14) morreram de pneumonia e possuíam mais de 12 meses de idade. Esta é uma enfermidade com prevalência variada no Brasil, sendo que animais de alta produção e/ou imunodeprimidos possuem uma maior susceptibilidade a infecção (Silva et al. 2005). Devido a intensidade de manejo em animais jovens, nesta faixa etária é mais comum se observar tétano (Almeida et al. 2012), fato que também foi observado neste trabalho, onde $72,73 \%$ (8/11) dos casos ocorreram em caprinos menores de 12 meses. A Fasciola hepatica é um dos principais parasitas de caprinos, sendo que no Sul do Brasil é o segundo maior causador de mortes parasitárias, ficando atrás apenas do $H$. contortus (Oliveira et al., 2008). Este dado também foi encontrado nesta pesquisa, onde 0 trematoda foi a segunda maior causa de morte parasitária, sendo a primeira haemoncose, e a quarta maior causa de óbito geral.

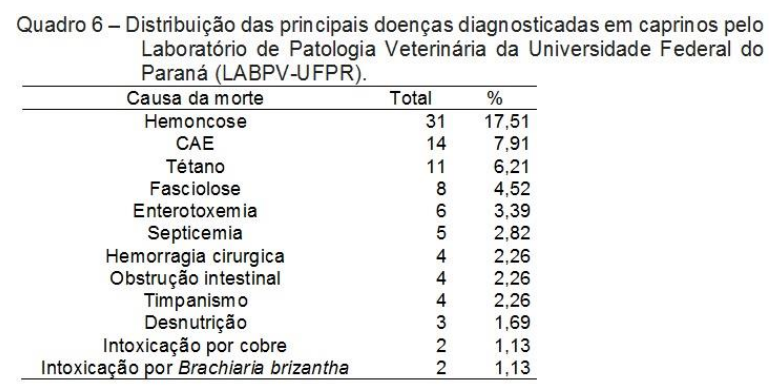

Doenças não listadas nas tabelas 2, 4 e 6, tiveram casos esporádicos e não possuem valor epidemiológico regional, sendo apenas achados patológicos individuais. Foram observados índices altos de diagnósticos inconclusivos, 10,87\% (70/644) em bovinos, 27,17\% (69/254) em ovinos e 28,81\% (51/177) em caprinos. Isto se ocorreu principalmente a alguns fatores como amostras autolisadas, ausência de lesões e coleta inadequada de material para envio ao laboratório. Estas também foram as 
causas mais comuns encontradas em pesquisa realizado em Santa Catarina (Casagrande et al. 2008). A falta de precisão de diagnóstico é variável em caso de pesquisa envolvendo envio de amostras por veterinários de campo, devido à falta de treinamento para analisar o animal e coletar amostras com relevância histopatológica (Lucena et al., 2010).

\section{CONCLUSÃO}

As principais causas de mortalidade em bovinos foram tuberculose, raiva, tristeza parasitária bovina e intoxicação por Pteridium aquilinum. Já para ovinos os motivos mais comuns foram haemoncose, enterotoxemia, intoxicação por Ipomoea asarifolia e tétano. Por fim, em caprinos as enfermidades dominantes foram haemoncose, CAE, tétano e fasciolose. Os dados presentes neste trabalho permitem que veterinários tenham acesso as principais enfermidades que acometem os ruminantes domésticos na Região Sudeste do Paraná.

\section{REFERÊNCIAS}

ALMEIDA, A. C. S.; RIBEIRO, M. G.; PAES, A. C.; MEGID, J.; OLIVEIRA, V. B.; FRANCO, M. $M$. J. Tetanus in small ruminants: retrospective study of major clinical and epidemiological findings in 11 cases. Arquivo Brasileiro de Medicina Veterinária e Zootecnia, v. 64, n.4, p. 1060-1064, 2012.

ALMEIDA, A. K.; MICHELS, I. L. O Brasil e a economia-mundo: o caso da carne bovina. Ensaios FEE, v.33, n.1, p.207-230, 2012.

ALMEIDA, M. B. D.; TORTELLI, F. P.; RIETCORREA, B.; FERREIRA, J. L. M.; SOARES, M. P.; FARIAS, N. A. R.; RIET-CORREA, F.; SCHILD, A. L. Tristeza parasitária bovina na região sul do Rio Grande do Sul: estudo retrospectivo de 1978-2005. Pesquisa Veterinária Brasileira, v.26, n.4, p.237-242, 2006.

ASSIS, T. S.; MEDEIROS, R. M. T.; RIETCORREA F.; GALIZA, G. J. N.; DANTAS, A. F.
M.; OLIVEIRA, D. M. Intoxicações por plantas diagnosticadas em ruminantes e equinos e estimativa das perdas econômicas na Paraíba. Pesquisa Veterinária Brasileira, v.30, n.1, p.1320, 2010.

BONNETT, B. N.; EGENVALL, A.; HEDHAMMAR, A.; OLSON, P. Mortality in over 350,000 insured Swedish dogs from 1995-2000: I. Breed-, gender-, age- and cause-specific rates. Acta Veterinay Scandinavica, v.46, p.105120, 2005.

BRASIL. Ministério da Agricultura Pecuária e Abastecimento. 2010. Boletim de Defesa Sanitária Animal. Casos de raiva em herbívoros e suínos no Brasil, no período anual de 1996 a $2010 . \quad$ Disponível em: http://casos\%20de\%20raiva\%20em\%20herb\%C $3 \%$ ADvoros $\% 20$ e $\% 20$ su\%C3\%ADnos\%20Brasil _por\%20UF\%20at\%C3\%A9\%202010\%20parcial .pdf. Acesso em: 23/08/2014.

CANNAS, A.; TEDESCHI, L. O.; FOX, D. G.; PELL, A. N.; VAN SOEST, P. J. A mechanistic model for predicting the nutrient requirements and feed biological values for sheep. Journal of Animal Science, v.82, n.1, p.149-169, 2004.

CASAGRANDE, R.A.; MAZZOCCO, M.B.; FRIZON, R.; LENTZ, D.; TRAVERSO, S.D.; GAVA A. 2008. Doenças de bovinos diagnosticadas pelo Laboratório de Patologia Animal CAV/UDESC de janeiro de 2000 a abril de 2008. Anais... Campo Grande: 3o Endivet, Campo Grande, 2008, p.55-56.

COSTA, V. M. M.; SIMÕES, S. V. D.; RIETCORREA, F. Controle das parasitoses

DIAS, J. A.; ALFIERI, A.; MÉDICI, K. C.; FREITAS, J. C.; NETO, J. S. F.; MÜLLER, E. E. Fatores de risco associados à infecção pelo herpesvírus bovino 1 em rebanhos bovinos da região Oeste do Estado do Paraná. Pesquisa Veterinária Brasileira, v.28, n.3, p.161-168, 2008.

DOS ANJOS, F. S.; CALDAS, N. V.; HIRAI, W. G. Mudanças nas práticas de autoconsumo dos produtores familiares: estudo de caso no sul do Brasil. Revista agroalimentaria, v.16, n.30, p.115-125, 2010.

DUTRA, S.; PEREIRA, F. B. Diagnóstico de situação das práticas de manejo sanitário em sistemas de produção de bovinos de corte. Veterinária e Zootecnia, v.19, n.4, p.522-530, 2012.

EZEBUIRO, O. G. C.; ABENGA, J. N.; EKEJINDU, G. O. C. The prevalence of trypanosome infection in trade cattle, goats and sheep slaughtered at the Kaduna abattoir. 
African Journal of Clinical and Experimental Microbiology, v.10, n.1, p.15-25, 2008.

FIGHERA, R. A. 2008. Causas de morte e razões para eutanásia em cães. Tese de Doutorado. Universidade Federal de Santa Maria, Santa Maria, RS. 171p.

FORTES, F. S.; KLOSTER, F. S.; SCHAFER, A. S.; BIER, D.; BUZATTI, A.; YOSHITANI, U. Y.; MOLENTO, M. B. Evaluation of resistance in a selected field strain of Haemonchus contortus to ivermectin and moxidectin using the Larval Migration on Agar Test. Pesquisa Veterinária Brasileira, v.33, p.2, p.183-187, 2013.

Gastrintestinais em ovinos e caprinos na região semiárida do Nordeste do Brasil. Pesquisa Veterinária Brasileira, v.31, n.1, p.65-71, 2011.

GONÇALVES, R. C.; SILVA, A. A.; FERREIRA, D. O. L.; CHIACCHIO, S. B.; LOPES, R. S.; BORGES, A. S.; Tristeza parasitária em bovinos na região de Botucatu - SP: estudo retrospectivo de 1986-2007. Semina Ciências Agrarárias, v.32, n.1, p.307-12.

GUEDES, K. M. R.; RIET-CORREA, F.; DANTAS, A. F. M.; SIMÕES, S. V. D.; NETO, E. G. M.; NOBRE, V. M. T.; MEDEIROS, R. M. T. Doenças do sistema nervoso central em caprinos e ovinos no semi-árido. Pesquisa Veterinária Brasileira, v.27, p.29-38, 2007.

LAYANA, J. E.; FERNANDEZ MIYAKAWA, M. E.; UZAL, F. A. Evaluation of different fluids for detection of Clostridium perfringens type D epsilon toxin in sheep with experimental enterotoxemia. Anaerobe, v.12, n.4, p.204-206, 2006.

LIMA, F. G.; GAGLIANI, L. H. Raiva: aspectos epidemiológicos, controle e diagnóstico laboratorial. UNILUS Ensino e Pesquisa, v.11, n.22, p.45-62, 2014.

LUCENA, R. B.; PIEREZAN, F.; KOMMERS, G. D.; IRIGOYEN, L. F.; FIGHERA, R. A.; BARROS, C. S. Doenças de bovinos no sul do Brasil: 6.706 casos. Pesquisa Veterinária Brasileira, v.30, n.5, p.428-434, 2010.

McGAVIN, M. D.; ZACHARY, J. F. Bases da patologia em veterinária. 4.ed. Rio de Janeiro: Elsevier, 2009. 1236p.

MOLENTO, M. B.; VERÍSSIMO, C. J.; AMARANTE, A. T.; VAN WYK, J. A.; CHAGAS, A. C. S.; ARAÚJO, J. V.; BORGES, F. A. Alternative techniques for the control of gastrointestinal nematodes in small ruminants. Arquivos do Instituto Biológico, v.80, n.2, p.253263, 2013.

MOORE, G. E.; BURKMAN, K.D.; CARTEN, M. N.; PETERSON M.R. Causes of death or reasons for euthanasia in military working dogs: 927 cases (1993-1996). Journal of American Veterinary Medical Association, v.219, p.209214, 2001.

OLIVEIRA, D. R.; FERREIRA, D. M.; STIVAL, C. C.; ROMERO, F.; CAVAGNOLLI, F.; KLOSS, A.; MOLENTO, M. B. Triclabendazole resistance involving Fasciola hepatica in sheep and goats during an outbreak in Almirante Tamandare, Parana, Brazil. Revista Brasileira de Parasitologia Veterinária, v. 17, p. 149-153, 2008.

PACHECO, A. M.; HAMZÈ, A. L.; AVANZA, M. F. B.; PEREIRA, D. M.; PEREIRA, R. E. P.; CIPRIANO, R. S.; LOT, R. F. S. Tuberculose bovina: relato de caso. Revista Científica Eletrônica de Medicina Veterinária, v.7, n.13, p.1-4, 2009.

PESSOA, C. R. M.; MEDEIROS, R. M. T.; RIETCORREA, F. Importância econômica, epidemiologia e controle das intoxicações por plantas no Brasil. Pesquisa Veterinária Brasileira, v.33, n.6, p.752-758, 2013.

PESSOA, C. R. M.; MEDEIROS, R.M.T.; RIETCORREA, F. Economic impact, epidemiology and control poisonous plants in Brazil. Pesquisa Veterinária Brasileira, v.33, n.6, p.752-758, 2013.

PONTES, R. V. R.; LIMA, M. S. Políticas de regionalização e condições favoráveis à competitividade: um estudo na cadeia agroindustrial de produção da carne bovina em município do norte do Brasil. Parcerias Estratégicas, v.17, n.34, p.45-64, 2013.

RIET-CORREA F.; BEZERRA C.W.C.; MEDEIROS R.M.T. Plantas Tóxicas do Nordeste. Sociedade Vicente Pallotti: Santa Maria, 2011. 82p.

RIET-CORREA, F.; MEDEIROS, R. M. Intoxicações por plantas em ruminantes no Brasil e no Uruguai: importância econômica, controle e riscos para a saúde pública. Pesquisa Veterinária Brasileira, v.21, n.1, p.38-42, 2001.

RISSI, D. R.; PIEREZAN, F.; OLIVEIRA FILHO, J. C.; FIGHERA, R. A.; IRIGOYEN, L. F.; KOMMERS, G. D.; BARROS, C. S. Doenças de ovinos da região Central do Rio Grande do Sul: 361 casos. Pesquisa Veterinária Brasileira, v.30, n.1, p.21-28, 2010.

ROSA, F. B.; GALIZA, G. J. N.; LUCENA, R. B.; SILVA, T. M.; CAPRIOLI, R. A.; BARROS, C. S. L.; KOMMERS, G. D. Doenças de caprinos diagnosticadas na região Central no Rio Grande do Sul: 114 casos. Pesquisa Veterinária Brasileira, v.33, n.2, p.199-204, 2013. 
SANTOS, R. L.; ALESSI, A. C. Patologia Veterinária. 1. ed. São Paulo: Roca, 2010. 423p.

SILVA, A. P. S. P.; SANTOS, D. V.; JUNIOR, I. K.; MACHADO, G.; HEIN, H. E.; VIDOR, A. C. M.; CORBELLINI, L. G. Ovinocultura do Rio Grande do Sul: descrição do sistema produtivo e dos principais aspectos sanitários e reprodutivos. Pesquisa Veterinária Brasileira, v.33, n.12, p.1453-1458, 2013.

SILVA, J. S.; CASTRO, R. S. D.; MELO, C. B. D.; FEIJÓ, F. M. C. Infecção pelo vírus da artrite encefalite caprina no Rio Grande do Norte. Arquivo Brasileiro de Medicina Veterinária, v.57, n.6, p.726-731, 2005.

SILVA, N. P.; FRANCISCO, A. C. Geração de energia elétrica a partir de dejetos suínos: um estudo de caso em uma propriedade rural na Região Oeste do Estado do Paraná. Nucleus, v.2, n.2, p.1-18, 2010.

SPRENGER, L. K.; AMARAL, C. H.; LEITE FILHO, R. V.; AGUIAR, T. N.; MOLENTO, M. B. Eficácia do fosfato de levamisol em nematódeos gastrintestinais de caprinos e ovinos. Archives of Veterinary Science, v.18, n.1, p.29-39, 2013.

TAPARO, V.; BERNARDES, J. O. R.; LOMBARDI, A. L.; MARINHO, M.; MENDES, L. C. N.; KANETO, C.; PERRI, S. H. V. Perfil de aglutininas anti-leptospira e anti-brucella e condições sanitárias de ovinos da região noroeste do estado de São Paulo,Brasil. Veterinária e Zootecnia, v. 19, n. 4, p. 593-600, 2012.

THRUSFIELD, M. Veterinary Epidemiology, 2. ed. Blackwell: Oxford, 1995. 483p.

TOKARNIA, C. H.; DÖBEREINER, J.; PEIXOTO, P. V. Plantas tóxicas do Brasil. Rio de Janeiro: Helianthus, 2000. 310p.

TORTELLI, F.P., RIET-CORREA B., FERREIRA J.L.M., SOARES M.P. \& SCHILD A.L. Babesiose cerebral na área de influência do Laboratório Regional de Diagnóstico. Boletim do Laboratório Regional de Diagnóstico, v.25, p.28-35, 2005.

WHO, Tuberculosis. Fact Sheet no. 104. World Health Organization, 2012. Disponível em: http://www.who.int. Acesso em: 15/08/2014. 